\title{
Rotation and Wolf-Rayet Star Formation
}

\author{
Georges Meynet
}

Geneva Observatory, CH-1290 Sauverny, Switzerland

\begin{abstract}
New results for $60 \mathrm{M}_{\odot}$ stellar models with different rotation rates are presented. Some effects of rotation on Wolf-Rayet star formation are discussed.
\end{abstract}

In these calculations we supposed that the angular momentum is locally conserved in radiative zones. The local conservation of angular momentum results from a balance between the outward flux of angular momentum essentially driven by shear instabilities and the inward flux resulting from meridional circulation. We checked by resolving the complete set of equations describing the transport of angular momentum (Maeder \& Zahn 1998) that this stationary state is reached in a relatively short timescale compared to the evolutionary timescale. This result may not hold for the whole stellar mass range (see Denissenkov et al. A\&A, in press).

The chemical species are diffused under the effects of both shear turbulence and meridional circulation. The diffusion coefficients used are given in Maeder (1997). We used the Ledoux criterion for setting the border of the convective core and, during the main sequence, we took into account semiconvective mixing. The effect of rotation on the mass loss rates is taken into account by using the Friend \& Abbott's (1986) formula.

Figure 1 presents the evolution of the structure of the star during the $\mathrm{H}$ and He-burning phases for two $60 \mathrm{M}_{\odot}$ models: one is non-rotating and the other is rotating with an initial angular velocity $\Omega$ equal to $60 \%$ the critical angular velocity $\Omega_{c}\left(\Omega_{c}\right.$ is the angular velocity at which the star begins to loose matter at the equator). Many differences can be seen :

1) The evolutionary scenarios are not the same. Before becoming a WR star, the non-rotating $60 \mathrm{M}_{\odot}$ model goes through a short LBV phase (see also Fig. 2) which intervenes after the $\mathrm{H}$ exhaustion in its core. When the hydrogen surface mass fraction becomes inferior to about 0.4, the star enters into the WR phase as a WNL star. In the case of the fast rotating model ${ }^{1}$, the star enters into the WR phase while still burning hydrogen in its core. The star thus skips the LBV phase and enters the WR phase at an earlier stage. Such an evolutionary scenario was also found by Fliegner \& Langer (1994).

2) The mechanism of WR star formation is different. Surface abundances characteristic of the WNL star appear in the rotating model, not as a result of the mass loss which uncovers core layers, but as a result of diffusive mixing

${ }^{1}$ Similar evolutions occur for $60 \mathrm{M}_{\odot}$ stellar models with $\Omega / \Omega_{c}=0.30$ and 0.40 and for $40 \mathrm{M}_{\odot}$ with $\Omega / \Omega_{c}=0.30,0.40$ and 0.60 . 


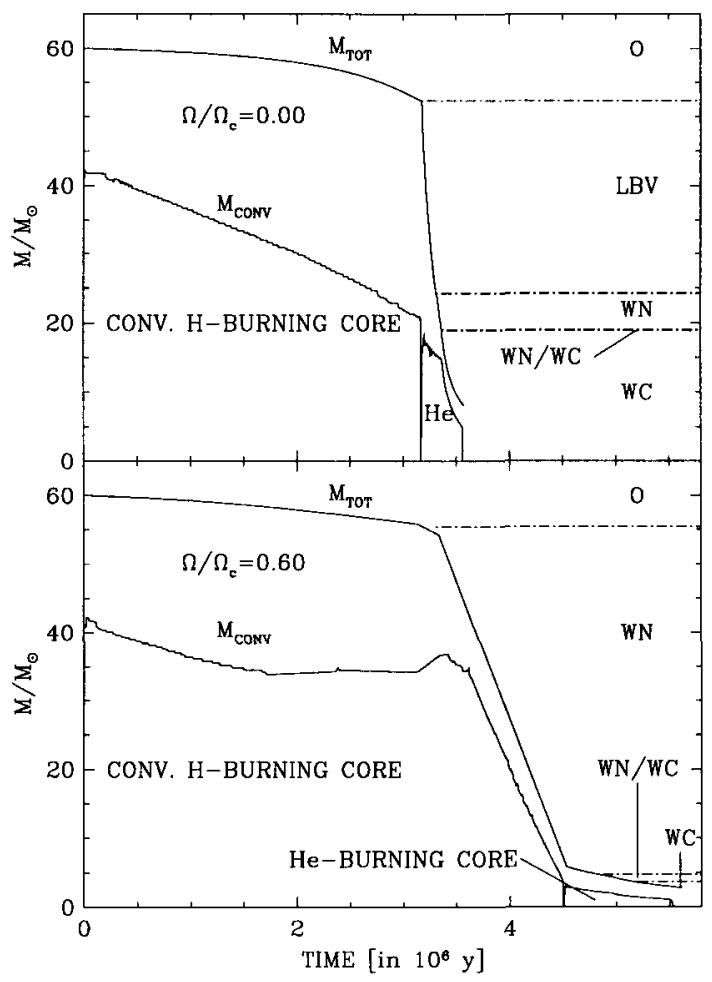

Fig. 1. Evolution of the total mass $M_{\mathrm{TOT}}$ and of the mass of the convective core $M_{\mathrm{CONV}}$ as a function of time. Various evolutionary stages are indicated on the right at the corresponding values of $M_{\mathrm{TOT}}$.

in the radiative zones. One observes that the same occurs for the entrance into the WC phase.

3) During the WN phase, the surface abundances are different. Indeed as a consequence of point 2) above, the $\mathrm{N} / \mathrm{C}, \mathrm{N} / \mathrm{O}$ ratios obtained at the surface of the rotating WN model have not yet reached nuclear equilibrium in contrast with the no rotation case where nuclear equilibrium is reached as soon as the star enters the WN phase.

4) The lifetimes are different. When $\Omega / \Omega_{c}$ increases the WR lifetime increases. The WN phase, as well as the transition WN/WC phase, become much longer. The WN/WC phase is characterized by the presence at the surface of both nitrogen and carbon. Non rotating models have difficulty in reproducing the observed number of such stars. Rotation might be the mechanism responsible for the formation of these stars (see also Langer 1991). 
The evolutionary tracks in the HR diagram are shown on Fig. 2. We can see that higher is the angular velocity, bluer are the tracks during the main sequence. This is a consequence of the greater efficiency of mixing at higher rotation. We can see also, that when the rotation rate increases, less luminous WC stars are produced. This is due to the fact that when the star rotates, it enters the WR phase at an earlier stage and thus begins to loose high amounts of mass early in its evolution. Therefore, the star enters the WC phase with a small mass and a low luminosity.

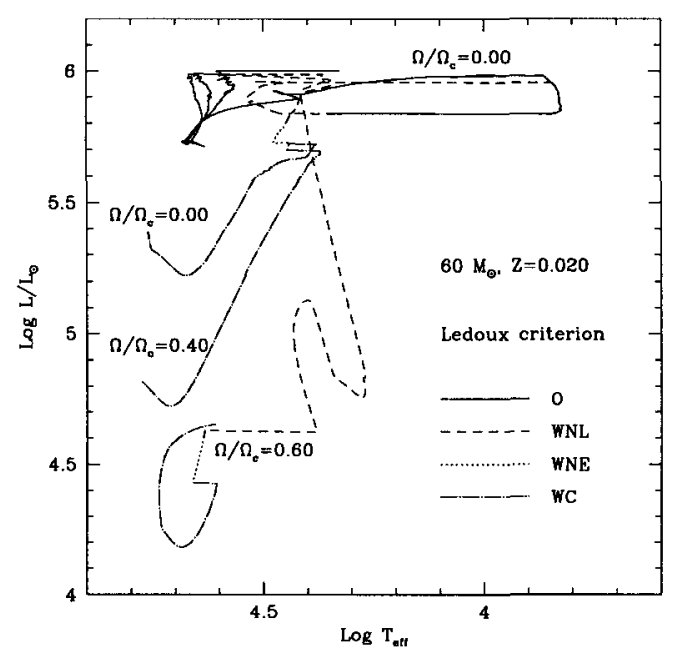

Fig. 2. Evolutionary tracks for $60 \mathrm{M}_{\odot}$ stellar models at solar metallicity for different rotational velocities. $\Omega / \Omega_{c}$ is the angular velocity on the ZAMS expressed as a fraction of the critical angular velocity $\Omega_{\mathrm{c}}$. On the main sequence, the higher the rotational velocity, the bluer is the track. Different WR subphases are indicated with different types of lines. Only part of the $\Omega / \Omega_{c}=0.30$ is shown.

\section{References}

Fliegner, J., Langer, N. (1994): IAU Symp. 163, p. 326

Friend, D.B., Abbott, D.C. (1986): ApJ 311, 701

Langer, N. (1991): A\&A 248, 531

Maeder, A. (1997): A\&A 321, 134

Maeder, A., Zahn, J.P., (1998): A\&A 334, 1000 


\section{Discussion}

R. Kudritzki: You are predicting a new lower mass limit for WR progenitors. I thought that there are clear observational limits on this mass. Are the new calculations in agreement with the observations?

G. Meynet: In fact, I do not propose a new lower mass limit, merely that some WR stars could originate from low-mass progenitors rotating very fast. But it remains to be estimated what proportion of low-mass stars rotate sufficiently rapidly for this scenario to occur. They certainly belong to the upper end of the rotational velocity distribution.

On the other hand, it must be added here that if we accept an observational lower initial mass limit for WR star formation of around, let's say, $25 \mathrm{M}_{\odot}$ at solar metallicity, it is difficult to account for this low value without enhanced mass-loss rates. It may be that rotation can help to explain this fact without enhanced mass loss, even if (due to clumpiness) the mass-loss rates are to be reduced.

S. Shore: In laboratory shear flows, the factor $1 / 4$ found from the classical Richardson instability criterion can be different; this could change the diffusion coefficient you have shown by a factor of 2 or more. So, what would such a change of " $\mathrm{D}$ " produce in your models? Also, if I understand correctly, Zahn's theory for sloping convection was developed in the perturbation limit; what happens at critical rotation? I would expect that the motion could be of high enough energy that the mechanical contribution, which is missing from the von Zeipel physical conditions, could be an important contributor to the energy balance at or near the equator.

A. Maeder: The factor $1 / 4$ is the usual classical value (e.g., Chandrasekhar 1961). If we were to assume that the excess energy is not $1 / 4(\mathrm{~d} U / \mathrm{d} z)^{2}$, but the same form with another numerical coefficient, the results would certainly be modified, like a shift in rotational velocities would do. Concerning von Zeipel's theorem, it applies to radiative envelopes. For stars with convective envelopes there is also some gravity darkening, but the results may be expected to show some quantitative differences. This is a problem which needs further investigation.

I. Appenzeller: Your models predict a significant fraction of H-rich WR stars. How does this compare with observations?

G. Meynet: H-rich WN stars are observed (see the paper by Hamann et al.). The interesting point to be checked is to estimate the extent to which the H-rich WN stars result from rotating progenitors. More rotating stellar models must be computed before careful comparisons with observations can be performed.

W.-R. Hamann: According to our spectral analysis (e.g., Hamann et al. 1995, A\&A 299, 151) late-subtype WN atmospheres have typical hydrogen mass fractions of $10-40 \%$. Thus it seems that these new evolutionary calculations are now in good agreement with the empirical abundances. 